NATIONAL LABORATORY

\title{
Characterization of Porosity Development in Oxidized Graphite using Automated Image Analysis Techniques
}

September 2009

\section{Prepared by}

Cristian I. Contescu

Senior Research Scientist

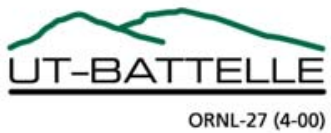




\title{
DOCUMENT AVAILABILITY
}

Reports produced after January 1, 1996, are generally available free via the U.S. Department of Energy (DOE) Information Bridge.

Web site http://www.osti.gov/bridge

Reports produced before January 1, 1996, may be purchased by members of the public from the following source.

\author{
National Technical Information Service \\ 5285 Port Royal Road \\ Springfield, VA 22161 \\ Telephone 703-605-6000 (1-800-553-6847) \\ TDD 703-487-4639 \\ Fax 703-605-6900 \\ E-mail info@ntis.gov \\ Web site http://www.ntis.gov/support/ordernowabout.htm
}

Reports are available to DOE employees, DOE contractors, Energy Technology Data Exchange (ETDE) representatives, and International Nuclear Information System (INIS) representatives from the following source.

Office of Scientific and Technical Information

P.O. Box 62

Oak Ridge, TN 37831

Telephone 865-576-8401

Fax 865-576-5728

E-mail reports@osti.gov

Web site http://www.osti.gov/contact.html

This report was prepared as an account of work sponsored by an agency of the United States Government. Neither the United States Government nor any agency thereof, nor any of their employees, makes any warranty, express or implied, or assumes any legal liability or responsibility for the accuracy, completeness, or usefulness of any information, apparatus, product, or process disclosed, or represents that its use would not infringe privately owned rights. Reference herein to any specific commercial product, process, or service by trade name, trademark, manufacturer, or otherwise, does not necessarily constitute or imply its endorsement, recommendation, or favoring by the United States Government or any agency thereof. The views and opinions of authors expressed herein do not necessarily state or reflect those of the United States Government or any agency thereof. 
ORNL/TM-2009/192

Next Generation Nuclear Plant (NGNP) Program

\title{
CHARACTERIZATION OF POROSITY DEVELOPMENT IN OXIDIZED GRAPHITE USING AUTOMATED IMAGE ANALYSIS TECHNIQUES
}

\author{
Cristian I Contescu \\ Timothy D Burchell
}

Date Published: September 2009

Prepared by

OAK RIDGE NATIONAL LABORATORY

Oak Ridge, Tennessee 37831-6283

managed by

UT-BATTELLE, LLC

for the

U.S. DEPARTMENT OF ENERGY

under contract DE-AC05-00OR22725 



\section{CONTENTS}

\section{Page}

LIST OF FIGURES

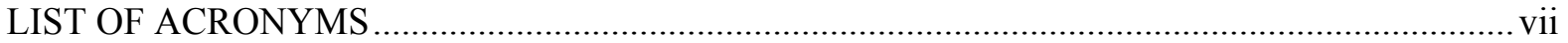

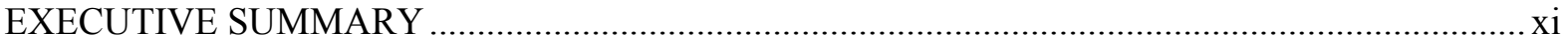

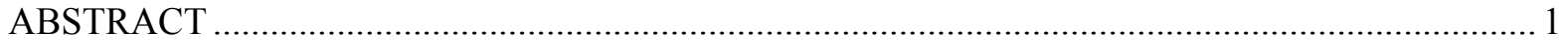

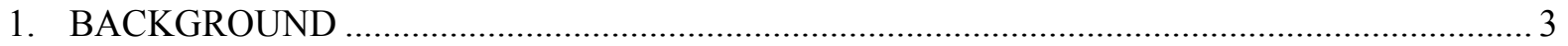

2. PREVIOUS RESULTS OF GRAPHITE OXIDATION RESEARCH AT ORNL ........................ 4

3. POROSITY DEVELOPMENT BY AUTOMATED IMAGE ANALYSIS TECHNIQUES .......... 5

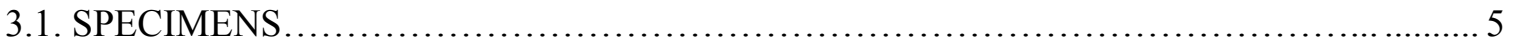

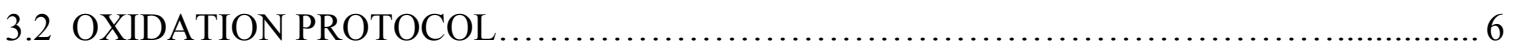

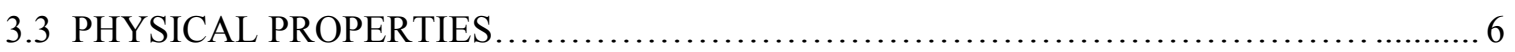

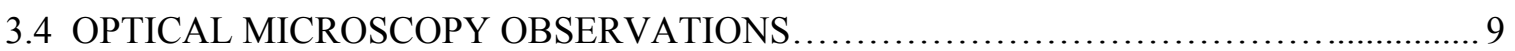

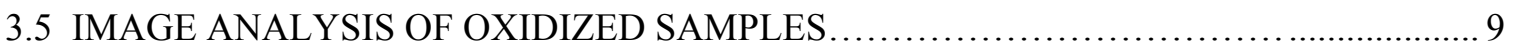

3.6 PRELIMINARY RESULTS FROM AUTOMATED IMAGE ANALYSIS .................. 11

4. DISCUSSION: OXIDATION EFFECTS ON MECHANICAL STRENGTH ............................ 20

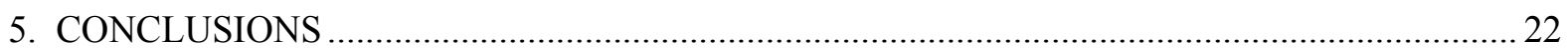

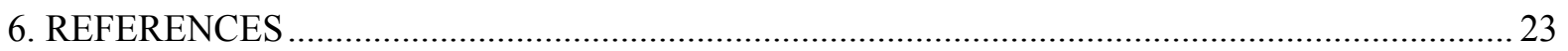





\section{LIST OF FIGURES}

Figure

Page

1 Cutting diagram of PCEA graphite block..... 5

2

Cutting diagram showing PCEA specimens used for oxidation studies ..... 6

Bulk density of oxidized PCEA specimens as a function of oxidation temperature and weight loss......

4 Effect of oxidation temperature on bulk density of graphite specimens oxidized at

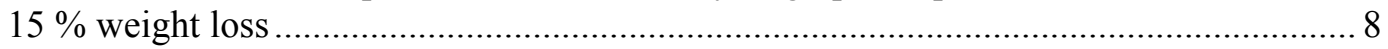

5

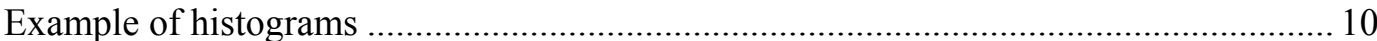

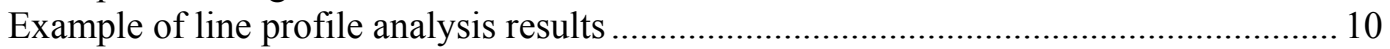

Mosaic image of PCEA graphite before oxidation .......................................................... 11

Mosaic image of PCEA graphite oxidized at $5 \%$ weight loss ....................................... 11

Mosaic image of PCEA graphite oxidized at $10 \%$ weight loss ..................................... 12

Mosaic image of PCEA graphite oxidized at $15 \%$ weight loss ...................................... 12

Mosaic image of PCEA graphite oxidized at $20 \%$ weight loss .................................... 13

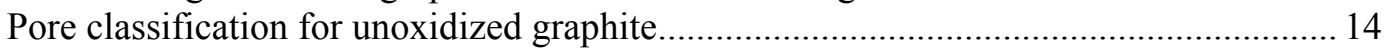

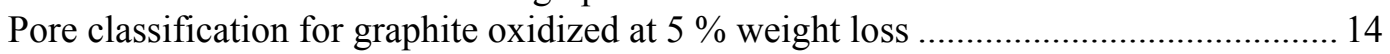

Pore classification for graphite oxidized at $10 \%$ weight loss ........................................ 15

Pore classification for graphite oxidized at $15 \%$ weight loss ........................................ 15

Pore classification for graphite oxidized at $20 \%$ weight loss ........................................ 16

Percentage distribution of pores in oxidized samples versus temperature and weight

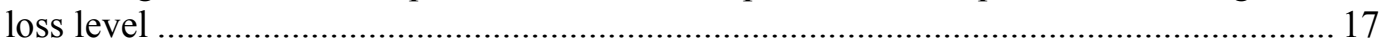

18 Pore density distribution in oxidized specimens versus temperature and weight loss

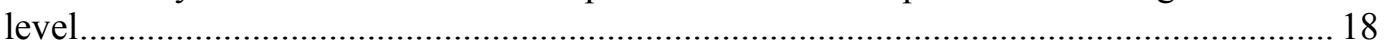

19 Nuclear graphite (NBG-18 grade) oxidized at $15 \%$ weight loss and different temperatures 


\section{LIST OF ACRONYMS}

$\begin{array}{ll}\text { AIA } & \text { automated image analysis } \\ \text { ASTM } & \text { American Society for Testing and Materials } \\ \text { GDMS } & \text { glow discharge mass spectrometry } \\ \text { high temperature gas-cooled reactor } \\ \text { HGTR } & \text { high temperature reactor } \\ \text { HTR } & \text { Korea Atomic Energy Research Institute } \\ \text { KAERI } & \text { Idaho National Laboratory } \\ \text { INL } & \text { Next Generation Nuclear Plant } \\ \text { NGNP } & \text { Oak Ridge National Laboratory } \\ \text { ORNL } & \text { parts per million } \\ \text { ppm } & \text { parts per million by volume } \\ \text { ppmv } & \end{array}$





\begin{abstract}
This document reports on initial activities at ORNL aimed at quantitative characterization of porosity development in oxidized graphite specimens using automated image analysis (AIA) techniques. A series of cylindrical shape specimens was machined from nuclear-grade graphite (type PCEA, from GrafTech International). The specimens were oxidized in air to various levels of weight loss (between 5 and $20 \%$ ) and at three oxidation temperatures $\left(600,650\right.$ and $\left.750{ }^{\circ} \mathrm{C}\right)$. The procedure used for specimen preparation and oxidation was based on ASTM D-7542-09. Oxidized specimens were sectioned, resin-mounted and polished for optical microscopy examination. Mosaic pictures of rectangular stripes $(25.5 \mathrm{~mm} \times 0.4 \mathrm{~mm})$ along a diameter of sectioned specimens were recorded. A commercial software (ImagePro) was evaluated for automated analysis of images. Because oxidized zones in graphite are less reflective in visible light than the pristine, unoxidized material, the microstructural changes induced by oxidation can easily be identified and analyzed. Oxidation at low temperatures contributes to development of numerous fine pores $\left(<100 \mu \mathrm{m}^{2}\right)$ distributed relatively uniformly over a certain depth $(5-6 \mathrm{~mm})$ from the surface of graphite specimens, while causing no apparent external damage to the specimens. In contrast, oxidation at high temperatures causes dimensional changes and substantial surface damage within a narrow band $(<1 \mathrm{~mm})$ near the exposed graphite surface, but leaves the interior of specimens with little or no changes in the pore structure. Based on these results it appears that weakening and degradation of mechanical properties of graphite materials produced by uniform oxidation at low temperatures is related to the massive development of fine pores in the oxidized zone. It was demonstrated that optical microscopy enhanced by AIA techniques allows accurate determination of oxidant penetration depth and of distribution of porosity in oxidized graphite materials.
\end{abstract}




\section{BACKGROUND}

The Next Generation Nuclear Plant (NGNP) will be a high-temperature gas-cooled reactor (HTGR) of modular construction, either prismatic or pebble-bed type. Graphite is a major structural component for the moderator and the core structures. Although all precautions are taken to ensure the purity of the cooling gas (helium), small quantities of oxidizing impurities (water vapor, oxygen) are unavoidable in the gas primary circuit. Although such impurities may never exceed the concentration of several parts per million by volume (ppmv), their long term oxidizing action and the effect on the integrity of structural graphite (and on the fuel matrix carbon) must be known.

It is known that the mechanical properties of graphite are severely reduced by oxidation in a variety of environments $[1,2,3]$. Graphite may suffer structural degradation via long term oxidation by impurity gases during normal operation conditions, or during uncontrolled oxidation in an air ingress accident leading to loss of coolant.

Low level of porosity is inherent to nuclear graphite; it results either from dimensional shrinking of graphite and coke, or from entrapment of gases released during thermal processing. As a microstructural component, graphite porosity plays an important role in the dynamics of fracture processes. On one hand, favorably oriented pores may be the place of crack initiation under low stress levels. On the other hand, crack propagation and growth may be influenced by the spatial relationship between large pores and pre-existing cracks. Although many types of pores, with various shape and size, are present in the graphite structure, it is generally agreed that large, slit-shaped pores are the most damaging to the graphite integrity [4]. Initiation of fracture cracks and propagation of cracks to sub-critical length before catastrophic failure are components of theoretical models of graphite fracture [5]. However, when a component of the graphite structure (of which the binder has the weakest oxidation resistance) is gasified through oxidation, it is conceivable that, along with density variations, the material's microstructure and basic strength properties would be affected $[6,7]$.

It is generally accepted that the dominant mechanism of graphite oxidation varies with the temperature [8]. Oxidation is controlled by the kinetics of the chemical process at low temperatures, but becomes diffusion-limited at high temperatures, and is strictly limited to mass transfer in the boundary layer at very high temperatures [9]. The temperature ranges specific to each of these mechanisms are not well defined [10] and are affected by sample's size and geometry [11]. When the rates of oxidation are slower than those of in-pore diffusion, oxidation is under kinetic control, and the oxidant penetrates deeper into the bulk of material, leading to (almost) uniform oxidation of specimens. Although with extremely slow rate, this mechanism of oxidation may cause extensive damage of material's strength, because it extends deep into the bulk of graphite. As the temperature goes higher and the rate of reaction accelerates, diffusion rate becomes the controlling factor and the penetration depth of the oxidant gradually diminishes. This leads to narrow oxidation zones limited to a thin layer at the graphite exposed surface, and leaves the bulk of material practically unchanged. Consequently, in spite of evident surface damage, the mechanical strength of the core material may not be seriously affected. 


\section{PREVIOUS RESULTS OF GRAPHITE OXIDATION RESEARCH AT ORNL}

During 2006-2009, ORNL led topical activities within ASTM International for establishing a new standard test method for characterization of air oxidation of graphite and manufactured carbon. The test method was drafted by ORNL based on a quality control procedure used by GrafTech International Ltd. The inter-laboratory tests, used for evaluation of the precision and bias, were conducted by ORNL and demonstrated good levels of statistical reproducibility and repeatability. The new test method was balloted and accepted both in the D02-F0 subcommittee and in the D02 committee of ASTM International, and has been given the designation D-7542-09: "Standard Test Method for Air Oxidation of Carbon and Graphite in the Kinetic Regime". The results of this test method help discriminate between carbon and graphite materials with different oxidation resistance. They can also be used for quality control purposes or for prediction of oxidation rates in air as a function of temperature, in conditions where oxidation is in the kinetic regime.

In parallel with the regulatory activity, research was initiated at ORNL on the mechanism of graphite oxidation, aimed at understanding the relationship between oxidation resistance and specific material properties of nuclear graphite.

Using direct density measurements (by layer peeling and physical measurements) the density profile of graphite specimens was measured after oxidation in air at various temperatures but at equal weight loss [10]. A larger than expected effect of oxidation conditions (temperature) was found on the density profile of oxidized samples. For specimens oxidized at low temperatures the oxidation zone extends some $10-12 \mathrm{~mm}$ deep from the surface, while at high temperatures it is very narrow $(<1$ $\mathrm{mm}$ ). Such differences cannot be detected by measurement of the overall oxidation rate. It was concluded that direct density measurements prove that the transition between homogeneous oxidation (kinetic regime) at low temperature and surface-limited oxidation (in-pore diffusion regime) is gradual with the increase in temperature. However, the direct method used for these density measurements was both very tedious and destructive.

In search of a non-destructive method for evaluation of density changes in oxidized graphite, researchers at ORNL have recently advanced a method based on acoustic microscopy combined with image analysis. The study was carried out in collaboration with Dr. S-H Chi of Korea Atomic Energy Research Institute (KAERI) during his sabbatical stage at ORNL in 2007-2008. The results were reported at the HTR 2008 conference (Washington, November 2008) and were published recently [12]. They demonstrate the validity of the method based on scanning acoustic microscopy with image processing for revealing density differences between oxidized zones in graphite. However, in order to increase the confidence level the method must be refined and supported by a larger number of examples.

In continuation of these efforts directed at modeling the oxidation process, an analytical procedure for calculation of penetration depth of the oxidant during transient oxidation conditions was recently proposed with help from Dr. Robert Wichner's external collaboration [13]. A short letter containing the results has been very recently accepted for publication [14]. Although based on several simplifying approximations, the analytical method predicts the development of non-uniform oxidation profiles by oxidation with water vapor or oxygen, their time evolution to a steady state condition, and the effect of temperature. The oxidation depths calculated in this simplified model were in fair agreement with our previously reported data from direct density measurements [10].

The remaining of this report presents the first results on development of porosity in oxidized graphite, obtained through optical microscopy enhanced with automated image analysis techniques. 


\section{POROSITY DEVELOPMENT BY AUTOMATED IMAGE ANALYSIS TECHNIQUES}

\subsection{SPECIMENS}

The material selected for analysis of porosity development was nuclear grade graphite (PCEA) from GrafTech International Ltd. A billet of PCEA graphite was purchased by ORNL. The billet was cut as shown in Figure 1. A slice (marked \# 2 in Fig. 1) in the center of the block was purified by gas extraction for removal of trace impurities. The ORNL contact for details on this material is Mr. Joseph Strizak.

The as-received material contained metal impurities ( $\mathrm{V}, \mathrm{Fe}, \mathrm{Ni}, \mathrm{Ti}, \mathrm{Ca}, \mathrm{Al})$ which needed to be removed because of their catalytic effect on oxidation. The test specimens used for oxidation studies reported here were machined from the gas-purified slice. After purification, the major impurities remaining in graphite were Si (1.5 ppm wt), S (4.6 ppm wt), P (0.22 ppm wt), V (0.28 ppm wt) and Mo $(0.35 \mathrm{ppm} w \mathrm{t})$. The impurity content is based on glow-discharge mass-spectrometric analysis (GDMS) done at Shiva Technologies in Syracuse NY.

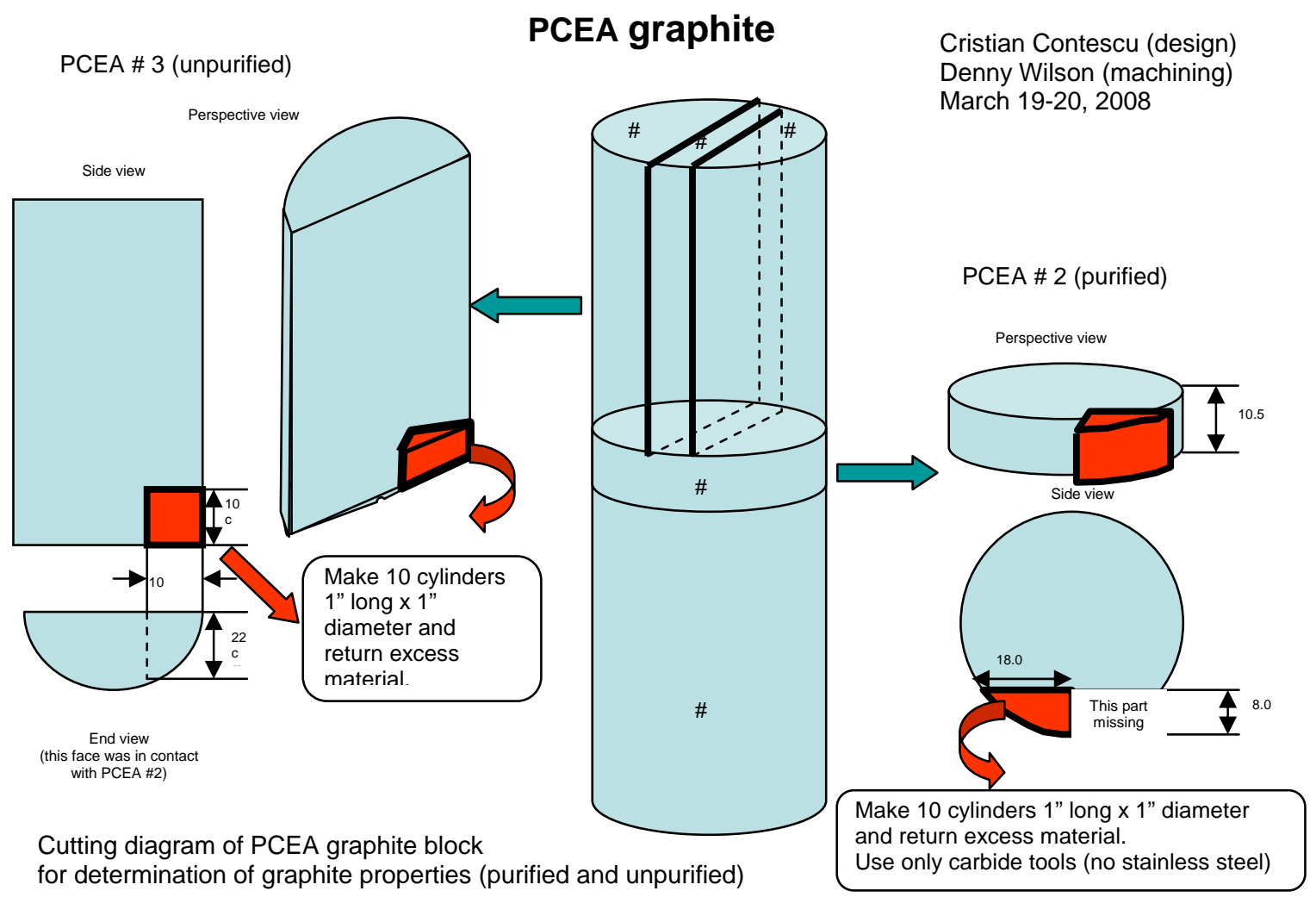

Fig. 1. Cutting diagram of PCEA graphite block. The specimens analyzed in this report originate from section marked PCEA \# 2 (purified by gas extraction). The red-colored sector was used for machining specimens for oxidation studies in 2008. The remaining part was used for machining specimens for oxidation and image analysis in 2009.

A number of specimens were machined in 2008 from a section of slice PCEA \# 2 (color-marked red in Fig. 1) and were used for characterization of graphite oxidation according to ASTM D-7542-09. More 
specimens were machined in 2009 from the remaining part of the same section of purified PCEA \#2. Figure 2 shows the cutting diagram of PCEA specimens used for oxidation studies and image analysis in 2009.

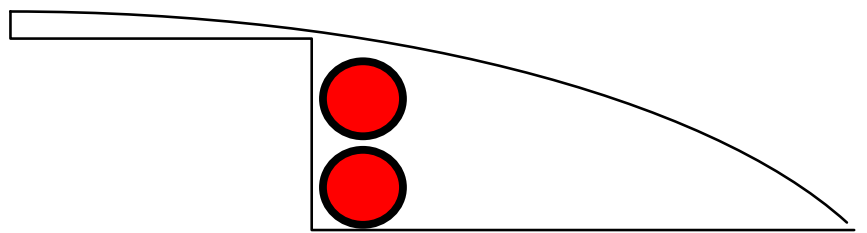

(a)

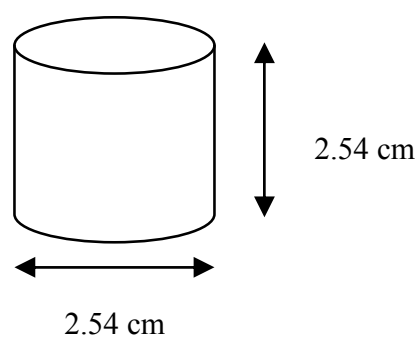

(b)

Fig. 2: Cutting diagram showing PCEA specimens used for oxidation studies (a) Cutting of the remaining part of PCEA \#2 graphite slice. This part was used for machining graphite specimens for oxidation and image analysis studies in 2009; (b) Specimen used for oxidation studies.

The graphite specimens used for oxidation studies were machined at ORNL according to specifications of ASTM D-7542-09. All test specimens were machined dry (no lubricants were used) with iron-free tools. All specimens have standard size and shape (cylinders, $25.4 \mathrm{~mm}$ diameter, 25.4 $\mathrm{mm}$ height) as shown in Fig. 2, b.

\subsection{OXIDATION PROTOCOL}

The specimens were oxidized in air using the protocol recommended by ASTM D-7542-09. The equipment used was the vertical furnace specially built at ORNL for conducting air oxidation studies. The same equipment was used to generate results for the inter-laboratory studies of this standard method. The procedure and the method of calculation are described in the research report of the interlaboratory study, available from ASTM International as RR: D02-1663. Further details on the ASTM method are available in ref. [15].

Three oxidation temperatures were selected for producing specimens: 600,650 and $750{ }^{\circ} \mathrm{C}$. At each temperature, specimens were prepared at four levels of oxidation, namely $5,10,15$, and $20 \%$ weight loss (compared to the initial weight before oxidation). A set of 12 specimens oxidized at various levels and at different temperatures was thus obtained for image analysis of oxidation effects on porosity.

In addition, a pristine (unoxidized) specimen from the same lot was used for comparison of the structure.

\subsection{PHYSICAL PROPERTIES}

Physical measurements were taken before and after oxidation (Table 1). The average bulk density of unoxidized PCEA specimens is $1.79 \mathrm{~g} / \mathrm{cm}^{3}$. The material loss caused by oxidation produces a decrease in bulk density of specimens. 
Table 1. Physical measurements data on PCEA specimens oxidized at different conditions

\begin{tabular}{|c|c|c|c|c|c|c|}
\hline Specimen ID & $\begin{array}{c}\text { Oxidation } \\
\text { Temperature } \\
\left({ }^{\circ} \mathrm{C}\right)\end{array}$ & $\begin{array}{c}\text { Weight } \\
\text { loss (\%) }\end{array}$ & Length (mm) & $\begin{array}{l}\text { Diameter } \\
\text { (mm) }\end{array}$ & Weight (g) & $\begin{array}{c}\text { Bulk } \\
\text { Density } \\
\left(\mathrm{g} / \mathrm{cm}^{3}\right)\end{array}$ \\
\hline PCEA-2 \# 15 & 600 & 5 & 25.41 & 25.41 & 21.9459 & 1.703 \\
\hline PCEA-2 \# 14 & 600 & 10 & 25.4 & 25.42 & 20.756 & 1.610 \\
\hline PCEA-2 \# 03 & 600 & 15 & 25.38 & 25.41 & 19.4873 & 1.514 \\
\hline PCEA-2 \# 04 & 600 & 15 & 25.48 & 25.42 & 19.7033 & 1.524 \\
\hline PCEA-2 \# 13 & 600 & 20 & 25.43 & 25.42 & 18.4242 & 1.428 \\
\hline PCEA-2 \# 10 & 650 & 5 & 25.41 & 25.41 & 21.7931 & 1.691 \\
\hline PCEA-2 \# 09 & 650 & 10 & 25.49 & 25.42 & 20.6289 & 1.595 \\
\hline PCEA-2 \# 01 & 650 & 15 & 25.39 & 25.41 & 19.3383 & 1.502 \\
\hline PCEA-2 \# 17 & 650 & 20 & 25.42 & 25.39 & 18.3275 & 1.424 \\
\hline PCEA-2 \# 05 & 700 & 15 & 25.44 & 25.31 & 19.264 & 1.505 \\
\hline PCEA-2 \# 06 & 700 & 15 & 25.38 & 25.36 & 19.1688 & 1.495 \\
\hline PCEA-2 \# 12 & 750 & 5 & 25.07 & 25.05 & 20.8429 & 1.687 \\
\hline PCEA-2 \# 11 & 750 & 10 & 24.95 & 24.95 & 19.5185 & 1.600 \\
\hline PCEA-2 \# 07 & 750 & 15 & 24.99 & 24.45 & 18.3891 & 1.567 \\
\hline PCEA-2 \# 16 & 750 & 20 & 24.91 & 23.93 & 17.2746 & 1.542 \\
\hline PCEA-2 \# 18 & none & none & 25.41 & 25.42 & 23.2461 & 1.803 \\
\hline
\end{tabular}

Selected information from Table 1 is represented in graphic format in Figures 3 and 4.

Figure 3 shows the variation of density with weight loss for graphite specimens oxidized at the three temperatures. The quasi-linear decrease in density after oxidation at 600 and $650{ }^{\circ} \mathrm{C}$ is consistent with uniform loss of material accompanied by minimal size variations. This is the evidence that density changes during oxidation were relatively uniformly distributed in the volume. On the contrary, for the specimens oxidized at $750{ }^{\circ} \mathrm{C}$, the leveling of density values (at levels higher than of other samples) between 5 and $20 \%$ weight loss show that oxidation was accompanied by corrosion of the external surface. Together with the dimensional changes recorded after oxidation, this result shows that the weight loss is limited to a very narrow surface layer for specimens oxidized at $750{ }^{\circ} \mathrm{C}$ (regardless of the degree of oxidation). Indeed, surface damage was visible for all specimens oxidized at $750{ }^{\circ} \mathrm{C}$, but was not detectable after oxidation at lower temperatures. 


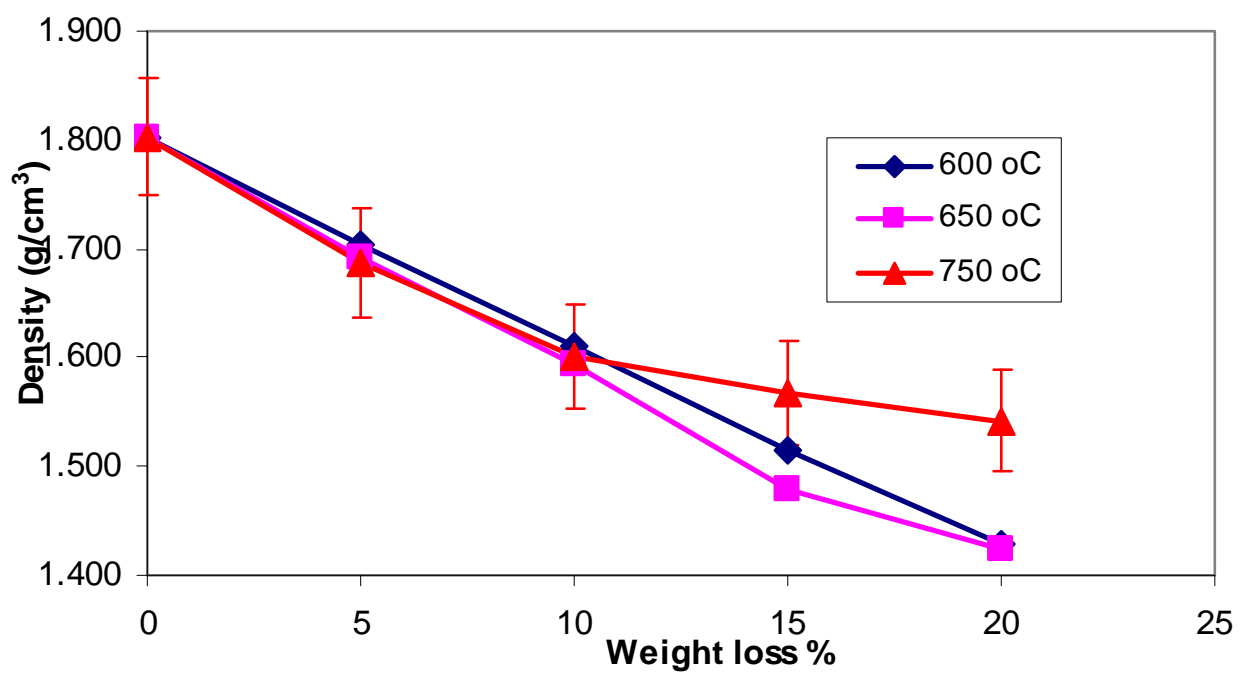

Fig. 3: Bulk density of oxidized specimens as a function of oxidation temperature and weight loss. The error bars ( $2 \%$ ) for samples oxidized at $750{ }^{\circ} \mathrm{C}$ reflect the uncertainty in size measurements for surfaceoxidized specimen, whose surface becomes very friable and corroded after oxidation.

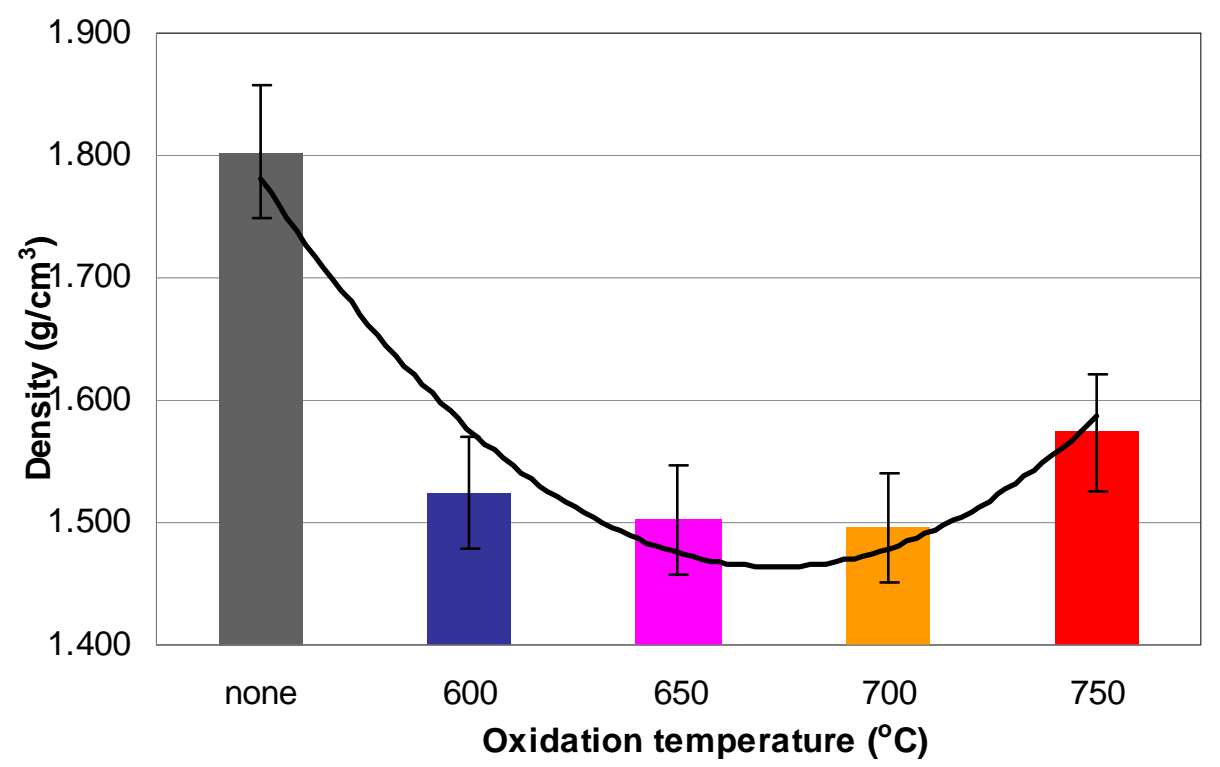

Fig. 4: Effect of oxidation temperature on bulk density of specimens oxidized at $15 \%$ weight loss. The error bars length is $2 \%$, as estimated for size measurements of surface-oxidized specimens.

Figure 4 shows the trend of density variation with oxidation temperature, at constant weight loss $(15 \%)$. The decrease in density after oxidation at 600 and $650{ }^{\circ} \mathrm{C}$ is consistent with material loss by 
oxidation accompanied by minimal variation of geometrical size or shape of specimens, as expected for homogeneous oxidation. This trend is reversed at $750{ }^{\circ} \mathrm{C}$. The slight increase in density for oxidation at 700 and $750{ }^{\circ} \mathrm{C}$ shows the increase in the fraction of unoxidized core of specimens, where the density change was minimal. At these temperatures, oxidation is accompanied by erosion of the outer surface, where most of the weight loss occurred.

\subsection{OPTICAL MICROSCOPY OBSERVATIONS}

For examination by optical microscopy, the specimens (both oxidized and unoxidized) were cut at about $1 / 3$ of their length. The shorter pieces were encased in resin and the inner surface exposed after cutting was polished using several grades (from coarse to fine) of alumina powder.

Several hundred optical photomicrographs were recorded at $200 \times$ magnification along the diameter of each specimen. A Nikon Microphot FXA (Japan) optical microscope was used. The microscope is equipped with motorized stage and autofocus control. The Leica Application Suite version 3.1.0 (Leica Microsystems CMS GmbHm Switzerland) was used for digital image acquisition. All individual images were recorded on a monochromatic 8 bit grey scale. In this scale a pixel with a value of 0 is the total black, and a pixel with value of 255 is the absolutely white. All intermediate pixel values describe different shades of grey, from dark grey (low pixel values) to light grey (large pixel values). Individual images were assembled in a rectangular mosaic image which covered an area of (usually) $25.5 \mathrm{~mm}$ x $4 \mathrm{~mm}$, along a diameter across the specimens $(25.4 \mathrm{~mm}$ before oxidation).

\subsection{IMAGE ANALYSIS OF OXIDIZED SAMPLES}

The mosaic images of oxidized graphite (and of a pristine sample for reference) were analyzed using the ImagePro Plus software version 6.3.0.512 (Media Cybernetics Inc.).

The first step of image analysis is spatial calibration, in order to set the measuring units of the system. A reference ruler was used for calibration, with traces separated by $0.1 \mathrm{~mm}$ and $0.02 \mathrm{~mm}$. This made it possible to work with real distances rather than conventional pixel units.

The next step was to enhance the contrast of each individual image in order to improve the ability of extracting objects. The automatic contrast enhancement function of ImagePro was used for consistency of results.

Next, the histogram analysis of each image was performed. Histograms illustrate the distribution of shades of grey in a graphical form, showing the frequency of pixel distribution along a scale of brightness intensities ranging from 0 (black) to 255 (white). The surface of optically polished graphite is in general reflective for visible light and appears bright, with correspondingly high pixel values. Porosity preexistent in graphite does not reflect visible light and is visible as grey (and sometimes black) zones. Similarly, the porosity and surface roughness created through oxidation appear as areas with low reflectivity, namely grey zones with lower than average pixel values.

Using histogram analysis of images collected before and after oxidation, or of images at various levels of oxidation, makes possible identification of effects introduced by oxidation, and segmentation of images based on objective criteria. The oxidized layer was easily identified visually as a somewhat darker zone extending a variable length from the edge of oxidized specimens. This allowed extraction of visual images of pores only, and analysis of pore sizes and spatial distribution along the specimen diameter. After analyzing and comparing numerous images, it was found that the modifications introduced by oxidation are essentially in the range of grey tones. These modifications were identified as peaks (or shoulders) on the histogram at pixel values below the median level. An example is shown in Figure 5, which compares histograms for a sample before oxidation and for samples oxidized at $20 \%$ weight loss and at three different temperatures. 

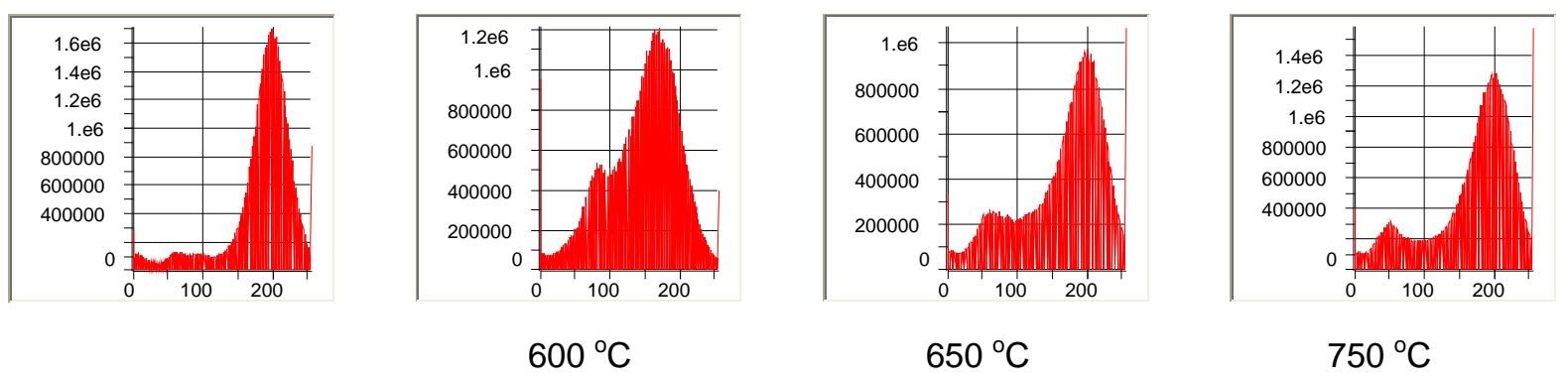

Fig. 5: Example of histograms. The abscissa shows grades of grey on the $0-255$ scale (black to white) and the ordinate axis shows the frequency of pixels distribution by levels of grey. Changes introduced by oxidation at $20 \%$ weight loss are seen as peaks or shoulders at pixel values below the median.

In addition, line profile analysis was used to collect actual pixel values in a line defined along the specimen's diameter. To lower the statistical noise, the line was "thickened" to a horizontal profile covering a large sample area (3500 - 4000 pixels). This analysis provides clear evidence of spatial distribution of grey areas (low pixel values associated with oxidized zones) near the edges of the specimen. In other words, line profile analysis is a measure of the degree of spatial uniformity of oxidation, which can be correlated with the penetration depth of oxidant under given oxidation conditions. An example of results of line profile analysis is shown in Figure 6 for the same set of samples as in Fig. 5. Before oxidation, the pixel values measured along the diameter of a $25.4 \mathrm{~mm}$ specimen were constantly near the 200 level, characteristic for the reflectivity of polished graphite. After oxidation, only some pixels in the center of the specimens are close to the 200 level. The oxidized zone, located near the edges, is about $1 \mathrm{~mm}$ thick after oxidation at $750{ }^{\circ} \mathrm{C}$, about $5 \mathrm{~mm}$ after oxidation at $650{ }^{\circ} \mathrm{C}$, and completely penetrates to the center of the specimen $(12.5 \mathrm{~mm})$ after oxidation at $600{ }^{\circ} \mathrm{C}$.

PCEA not oxidized

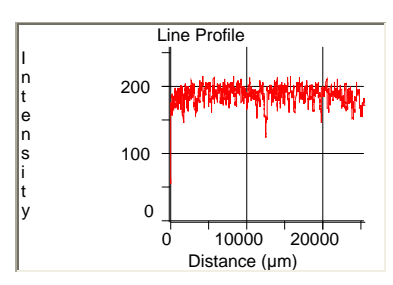

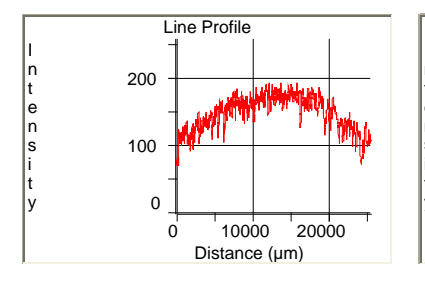

$600{ }^{\circ} \mathrm{C}$

\section{PCEA oxidized at $20 \%$ weight loss}

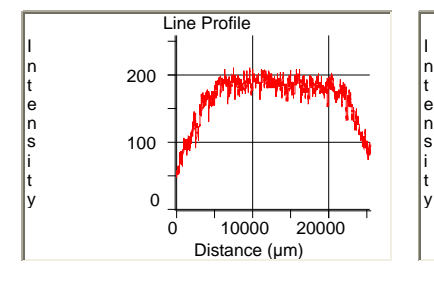

$650{ }^{\circ} \mathrm{C}$

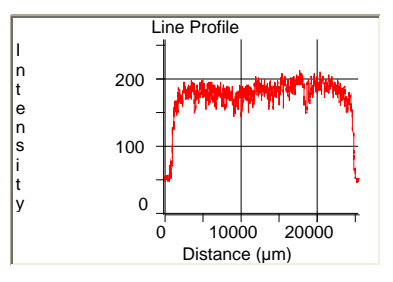

$750{ }^{\circ} \mathrm{C}$

Fig. 6: Example of line profile analysis results. The temperature dependence of the oxidation profile is compared at $20 \%$ weight loss. The ordinate represents average pixel intensity values (levels of grey) on the $0-255$ (black to white) scale. The abscissa is the distance (in) along the specimen diameter. The diameter of unxidized specimens was $25.4 \mathrm{~mm}(25,400 \mu \mathrm{m})$. 
Next, the images were segmented based on histogram analysis in order to extract objects associated with porosity introduced by oxidation. The histograms were cut at an upper level of grey intensities located just before the peak of bright intensities caused by the normal reflectivity of polished graphite (Fig. 5). Segmentation of histograms was made manually, with slight adjustments between samples, but in general the upper limit selected was in the range of pixel values $120-140$.

The objects found in the selected range of grey intensities were then counted and measured. The measurements comprised area, perimeter, and aspect ratio. The minimum pixel size for the microscope images had an area of $41 \mu \mathrm{m}^{2}$. This was the minimum object (pore) area that could be identified.

\subsection{PRELIMINARY RESULTS FROM AUTOMATED IMAGE ANALYSIS}

Figure 7 shows mosaic images of pristine (not oxidized) graphite. Figures $8-11$ show similar images for specimens oxidized at four different levels of oxidation (5 to $20 \%$ weight loss) and three different temperatures.

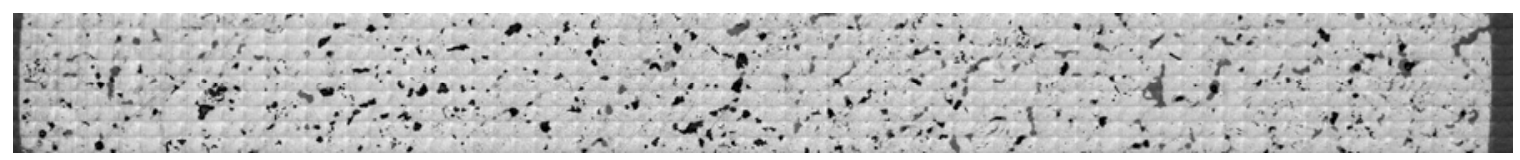

PCEA not oxidized (sample \# 18)

Fig. 7: Mosaic image of PCEA graphite before oxidation (magnification $200 \mathrm{x}$ )

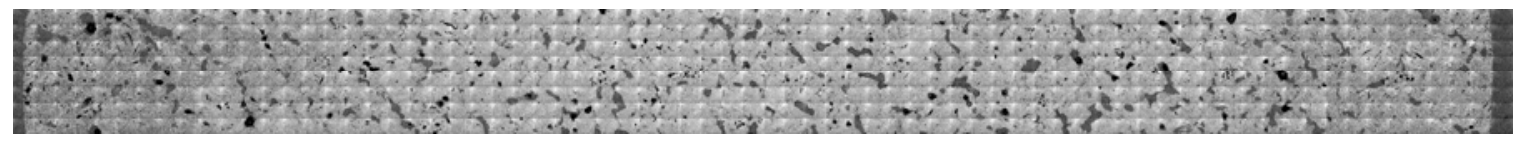

$5 \%$ weight loss at $600{ }^{\circ} \mathrm{C}$ (sample \# 15)

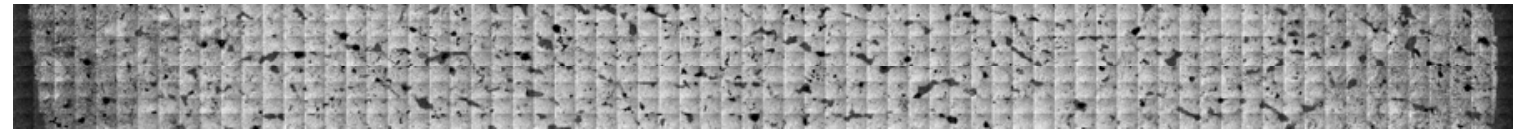

$5 \%$ weight loss at $650{ }^{\circ} \mathrm{C}$ (sample \# 10)

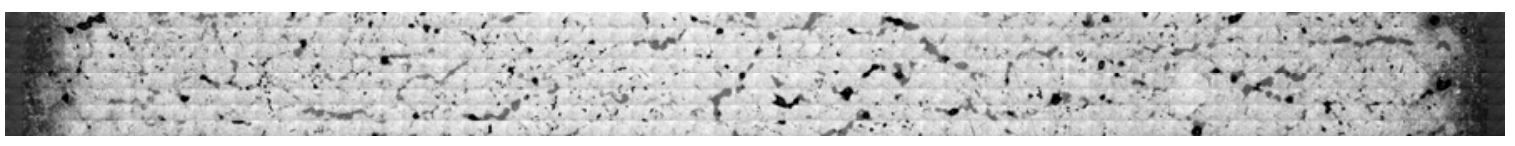

$5 \%$ weight loss at $750{ }^{\circ} \mathrm{C}$ (sample \# 12)

Fig. 8: Mosaic images of PCEA graphite oxidized at $5 \%$ weight loss (magnification $200 \mathrm{x}$ ) 


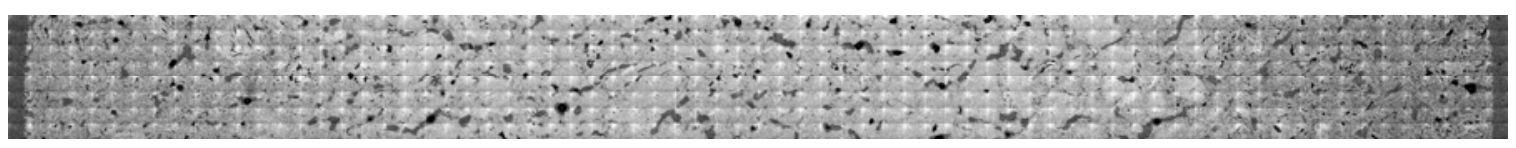

$10 \%$ weight loss at $600{ }^{\circ} \mathrm{C}$ (sample \# 14)

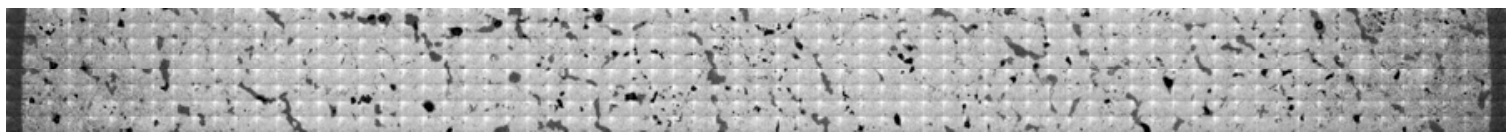

$10 \%$ weight loss at $650{ }^{\circ} \mathrm{C}$ (sample \# 09)

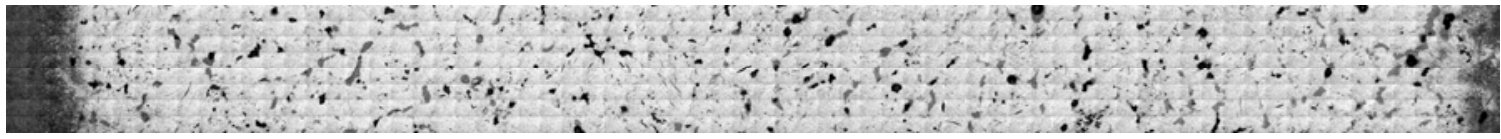

$10 \%$ weight loss at $750{ }^{\circ} \mathrm{C}$ (sample \# 11)

Fig. 9: Mosaic images of PCEA graphite oxidized at $10 \%$ weight loss (magnification $200 \mathrm{x}$ )

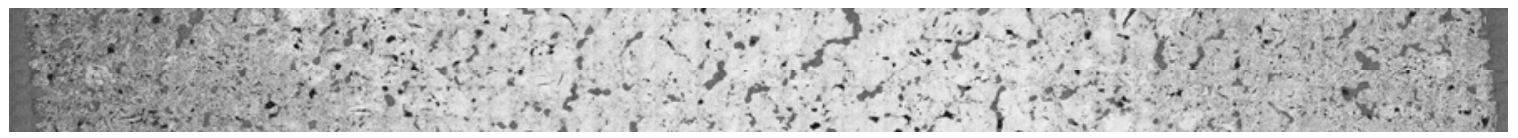

$15 \%$ weight loss at $600{ }^{\circ} \mathrm{C}$ (sample \# 03)

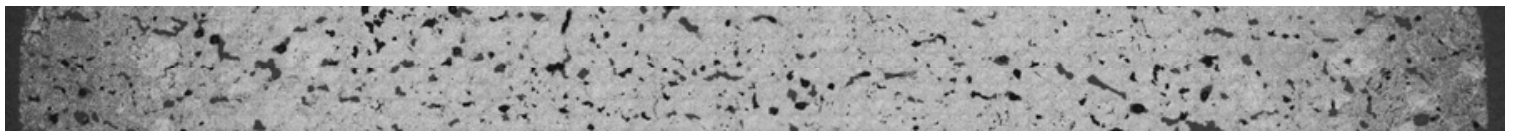

$15 \%$ weight loss at $650^{\circ} \mathrm{C}$ (sample \# 01)

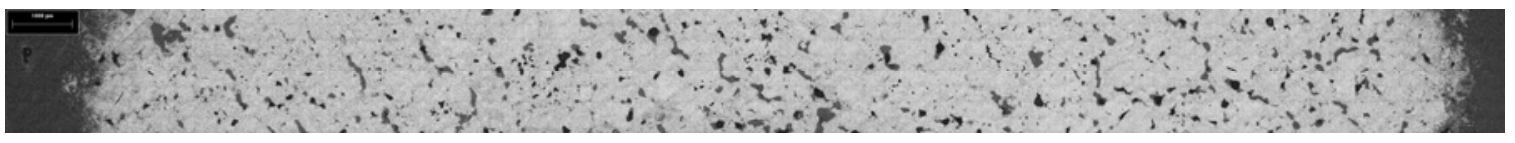

$15 \%$ weight loss at $750{ }^{\circ} \mathrm{C}$ (sample \# 07)

Fig. 10: Mosaic images of PCEA graphite oxidized at $15 \%$ weight loss (magnification $200 \mathrm{x}$ ) 


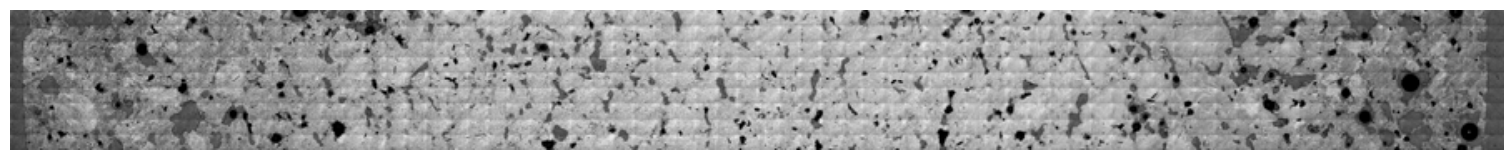

$20 \%$ weight loss at $600{ }^{\circ} \mathrm{C}$ (sample \# 13)

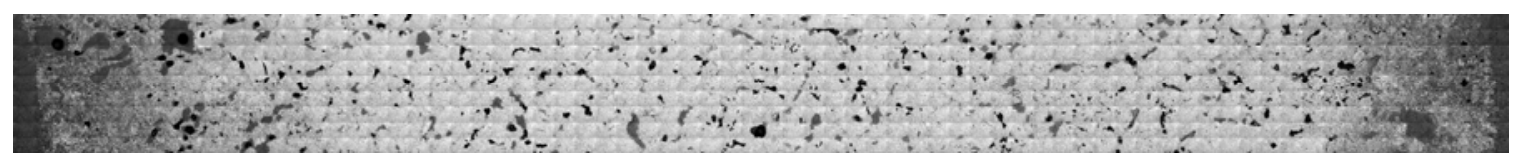

$20 \%$ weight loss at $650{ }^{\circ} \mathrm{C}$ (sample \# 17)

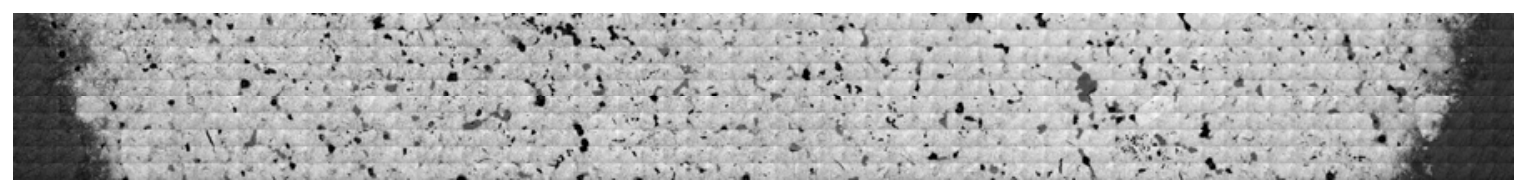

$20 \%$ weight loss at $750{ }^{\circ} \mathrm{C}$ (sample \# 16)

Fig. 11: Mosaic images of PCEA graphite oxidized at $20 \%$ weight loss (magnification $200 \mathrm{x}$ )

In the next analysis step, the pores created by oxidation were counted and classified. The histogram was segmented and the peak(s) corresponding to grey areas representing pores were selected. This procedure isolates less reflective objects, actually portions of the graphite surface associated with various types of porosity (both intrinsic to graphite structure and introduced by oxidation). The objects isolated in the preceding step were then counted and measured. The total number of objects selected by this method varied between 20,000 for pristine graphite and 70,000 for specimens heavily oxidized at low temperatures. Because the selection also included some large dark areas from the resin exposed to the exterior of graphite specimens, the latter objects were identified and eliminated. The remaining objects were then analyzed statistically and classified by their area. It was found that a classification in three groups (Table 2) is both meaningful and fast. The three groups (Table 2) were marked with different colors in the mosaic pictures shown in Figures $12-16$. The most numerous class is color-coded pink in these figures and comprises fine pores (mean area 57 $\mu \mathrm{m}^{2}$ ), which represent $85-90 \%$ of the total number of objects (pores) for all specimens analyzed. In the second class, color-coded blue, are grouped objects (pores) with a mean area of $780 \mu \mathrm{m}^{2}$, and representing about $10-15 \%$ of the total number. Lastly, a third class (color-coded green) consists of a very small number of very large objects (mean area 56,000 $\mathrm{m}^{2}$ ) which represent $<1 \%$ of the total. The line profiles and the histograms corresponding to each image are also shown in next figures. The scale bar length is $1 \mathrm{~mm}$.

Table 2: Classes of pores (by pore area) and their properties

\begin{tabular}{lccc}
\hline \multirow{2}{*}{ Pore classification (rounded values) } & 1 & 2 & 3 \\
\cline { 2 - 4 } & "fine" & "medium" & "very large' \\
\hline \hline Mean Pore Area $\left(\mathrm{m}^{2}\right)$ & 60 & 780 & 56000 \\
Mean Aspect Ratio & 1.3 & 2.3 & 2.2 \\
\hline
\end{tabular}




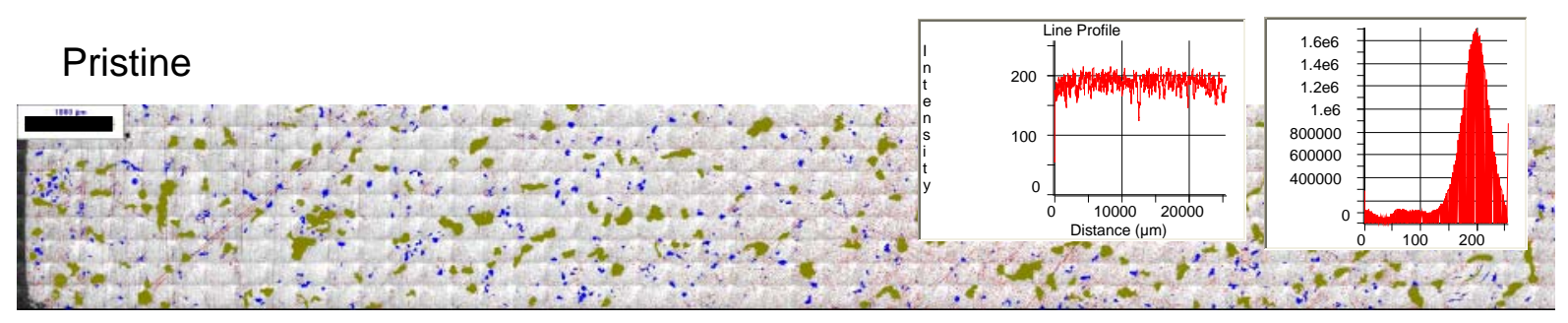

Fig. 12: Pore classification for unoxidized graphite (scale bar length $1 \mathrm{~mm}$ ). Line profile information (pixel values vs. distance in $\mu \mathrm{m}$ ) and histogram (population frequency by pixel values) information is overlapped.

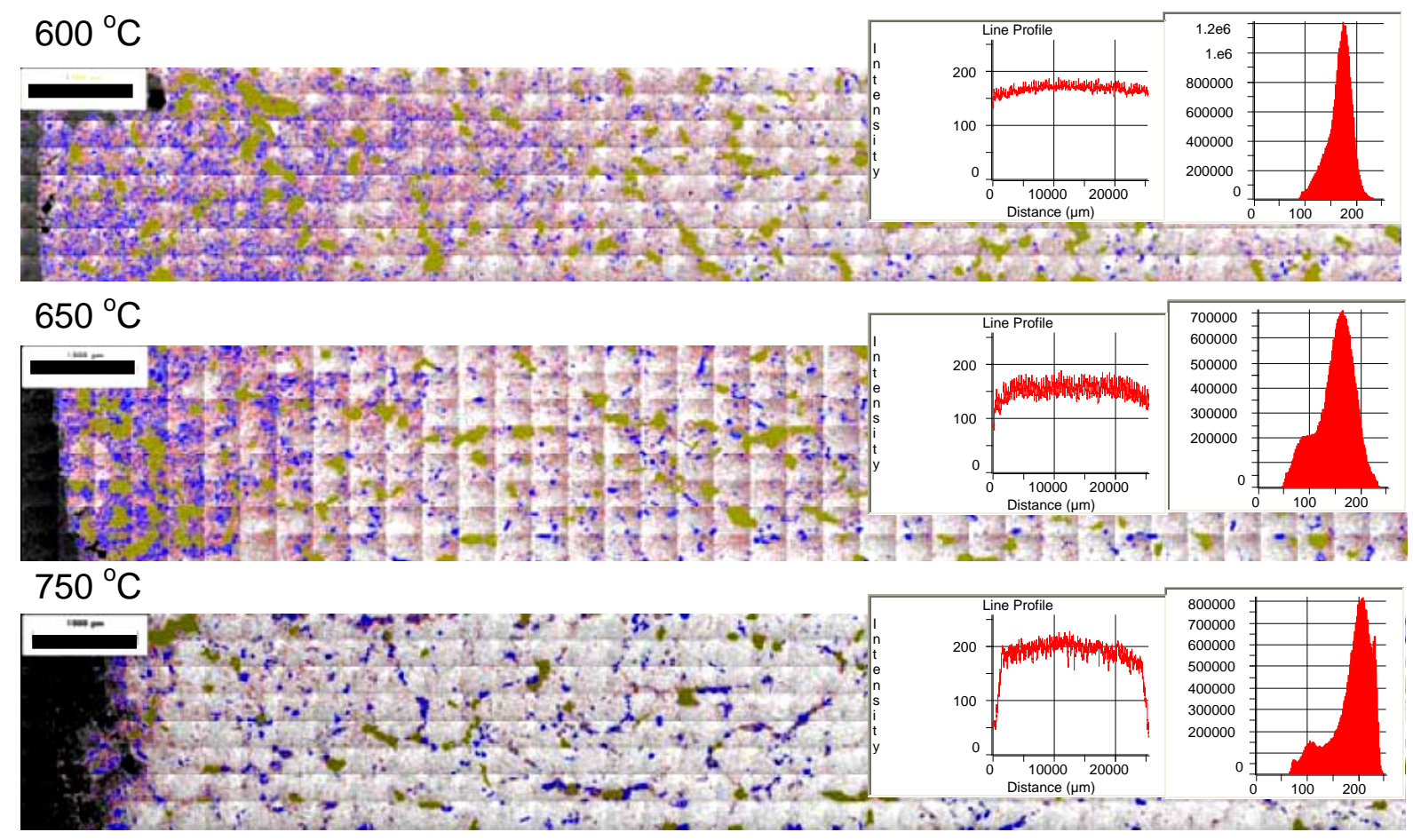

Fig. 13: Pore classification for graphite oxidized at $5 \%$ weight loss (scale bar length $1 \mathrm{~mm}$ ). Line profile information (pixel values vs. distance in $\mu \mathrm{m}$ ) and histogram (population frequency by pixel values) information is overlapped. 


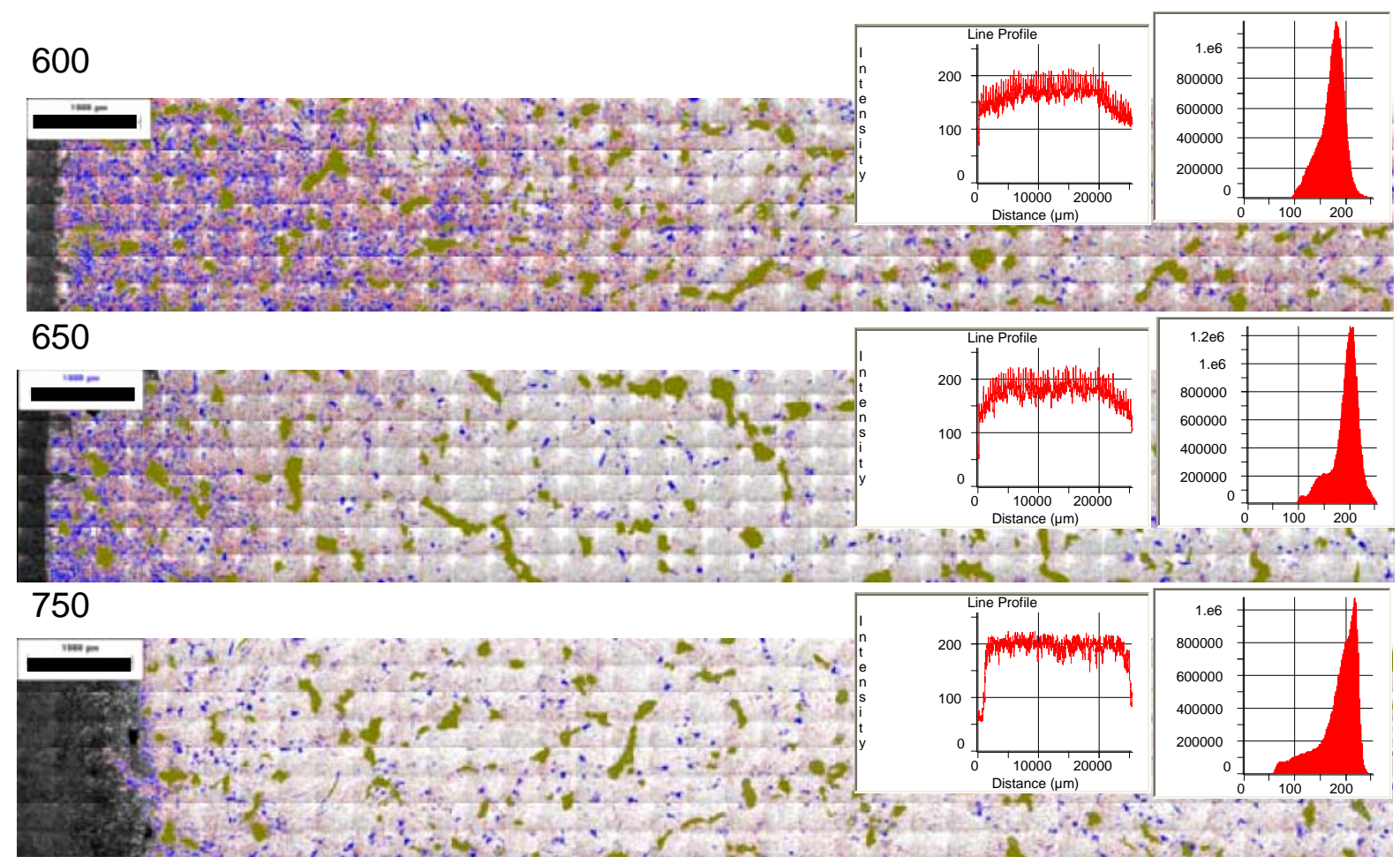

Fig. 14: Pore classification for graphite oxidized at $10 \%$ weight loss (scale bar length $1 \mathrm{~mm}$ ). Line profile information (pixel values vs. distance in $\mu \mathrm{m}$ ) and histogram (population frequency by pixel values) information is overlapped.
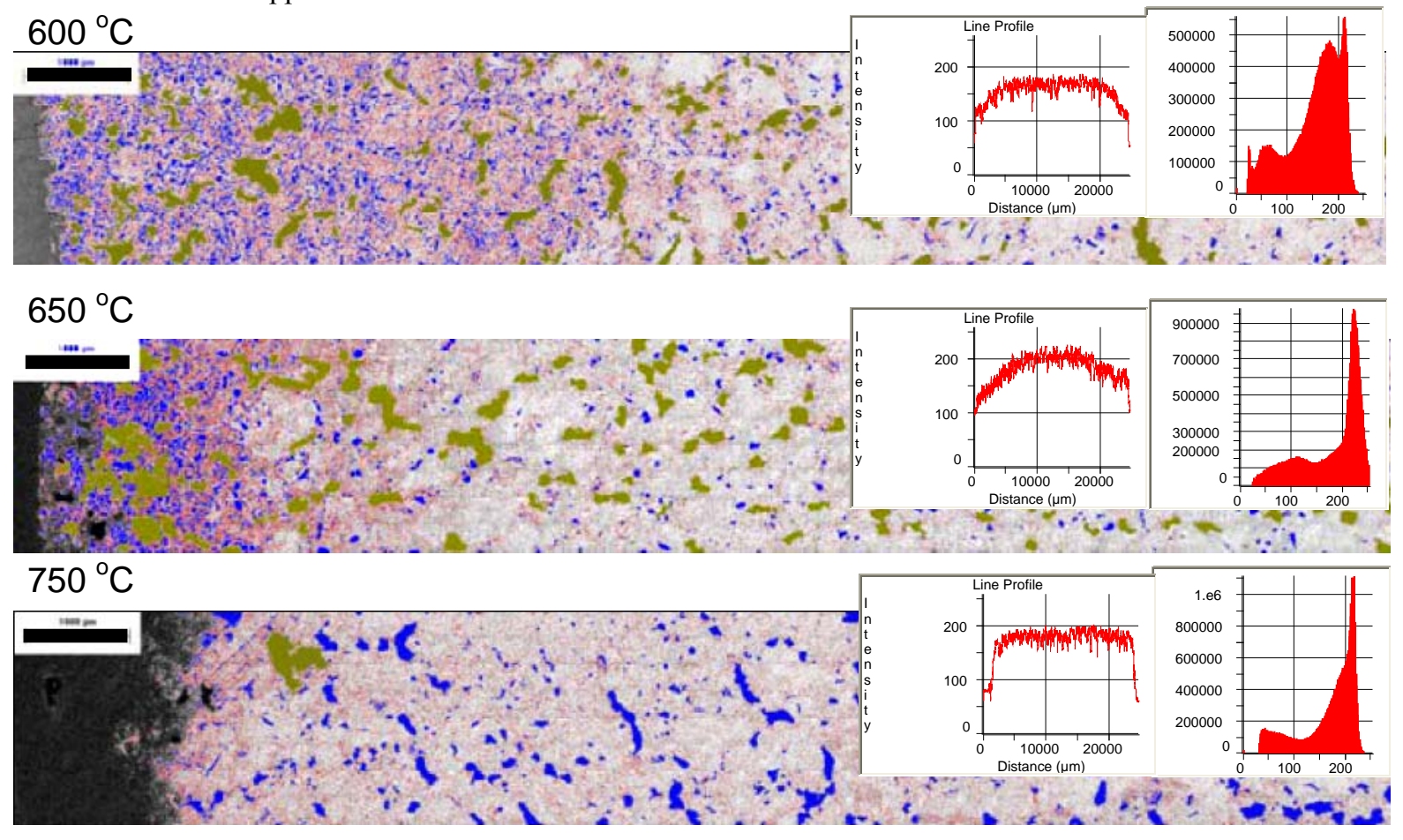

Fig. 15: Pore classification for graphite oxidized at $15 \%$ weight loss (scale bar length $1 \mathrm{~mm}$ ). Line profile information (pixel values vs. distance in $\mu \mathrm{m}$ ) and histogram (population frequency by pixel values) information is overlapped. 

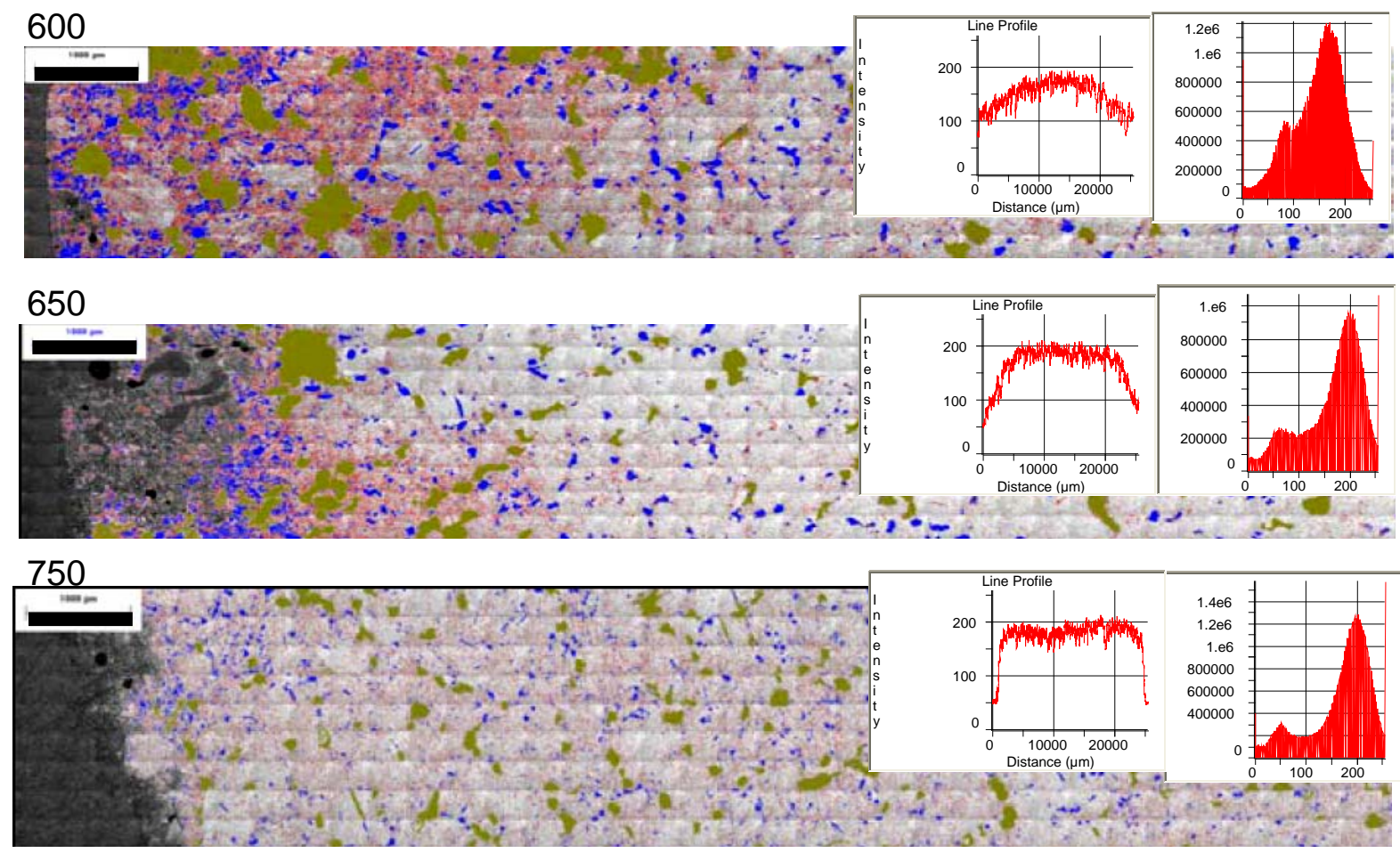

Fig. 16: Pore classification for graphite oxidized at $20 \%$ weight loss (scale bar length $1 \mathrm{~mm}$ ). Line profile information (pixel values vs. distance in $\mu \mathrm{m}$ ) and histogram (population frequency by pixel values) information is overlapped.

Examination of Figures $12-16$ shows the following main trends:

- First, oxidation seems not to enlarge or multiply the pores already present in the graphite structure. These pores are large in size and originate from shrinking of graphite and coke or from gas entrapment during thermal treatment.

- Second, oxidation is accompanied by development of numerous fine pores, located preferentially at the edges of oxidized specimens, i.e. in the areas previously identified as penetrated by the oxidant. The number of fine pores, which are present in a small amount in the pristine sample, increases dramatically in the oxidized samples.

- For samples oxidized at low temperatures $\left(600-650{ }^{\circ} \mathrm{C}\right)$, the zone with high density of fine pores extends deeply in the volume of the specimen. For these samples, some gradient in the density of fine pores is visible over distances as large as 5-6 $\mathrm{mm}$. The depth of oxidized layer is narrower at $650{ }^{\circ} \mathrm{C}$ (compared to $600{ }^{\circ} \mathrm{C}$ ) and narrows even more $(<1 \mathrm{~mm})$ at $750{ }^{\circ} \mathrm{C}$. For the latter, the specimen edge becomes very irregular and rough.

The next two figures show statistical information on the pore groups identified on oxidized specimens. In Figure 17 the distribution of pores between the three classes is plotted as percentage of the total number of pores. In all samples, the fine pores (class 1) dominate the porosity with more than $80 \%$ of total number, followed by medium size pores (class 2 ) which represent $10-15 \%$; the very large pores (class 3 ) represent under $1 \%$ of total. In this analysis, the differences between the pristine sample and oxidized samples are minor: in most cases, oxidation causes a slight increase in the percentage of medium size pores (class 2) at the expense of fine pores (class 1). 


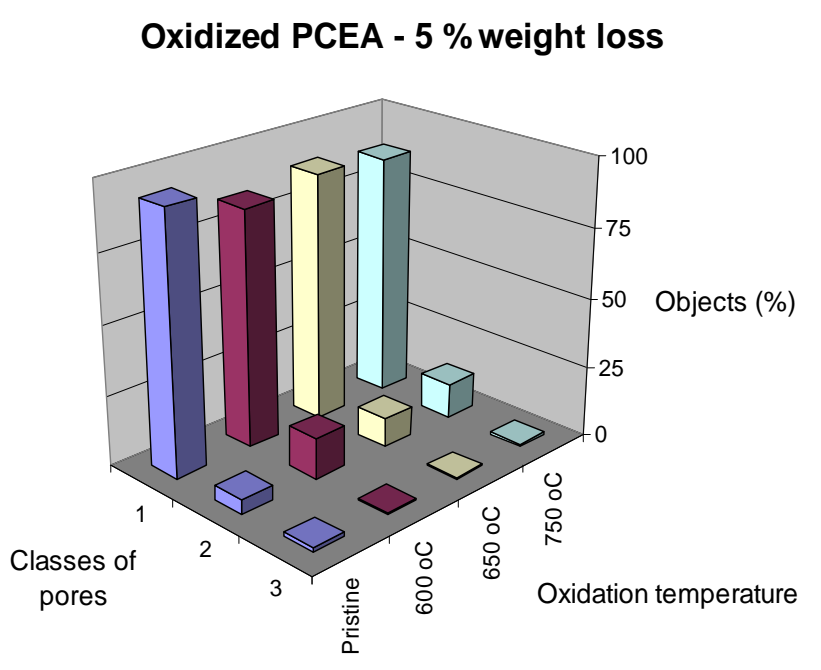

Oxidized PCEA - $15 \%$ weight loss

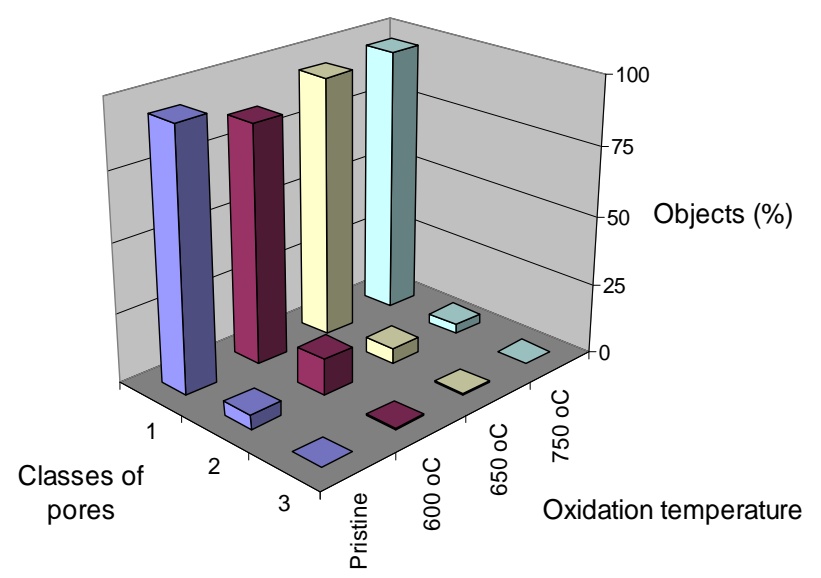

Oxidized PCEA - $10 \%$ weight loss

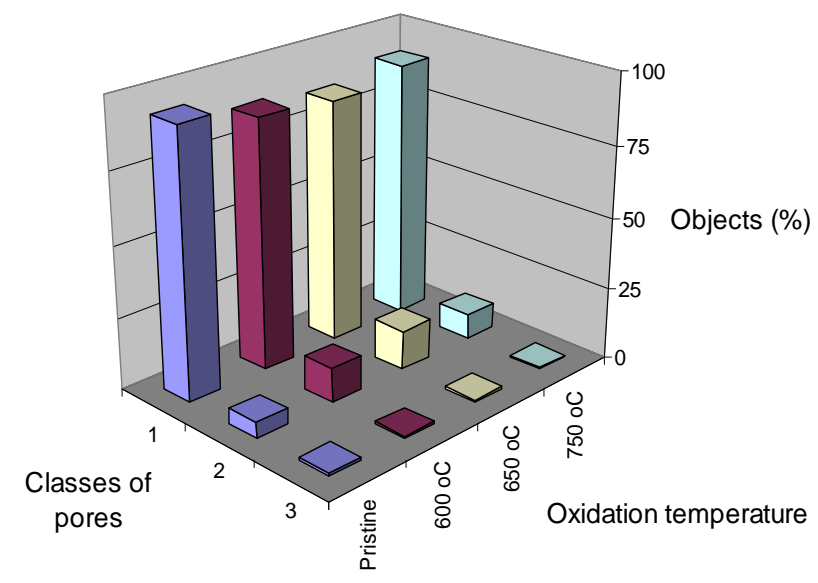

Oxidized PCEA - 20 \% weight loss

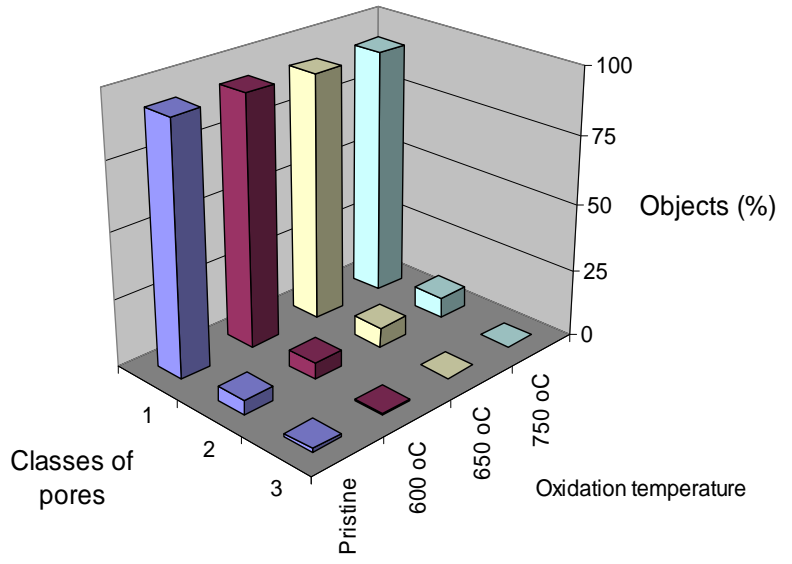

Fig.17: Percentage distribution of pores in oxidized samples versus temperature and weight loss level. Classes of pores are defined in Table 2. 


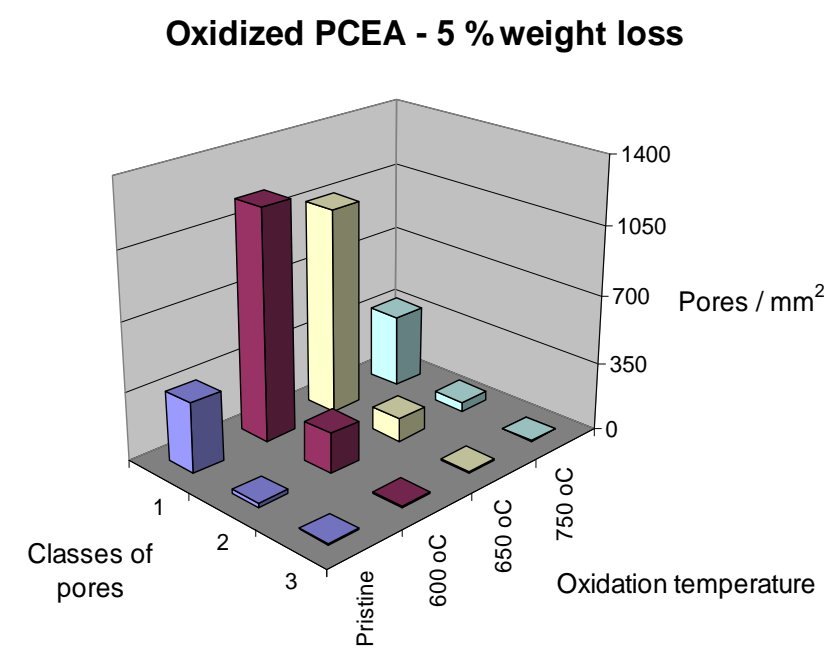

Oxidized PCEA - $15 \%$ weight loss

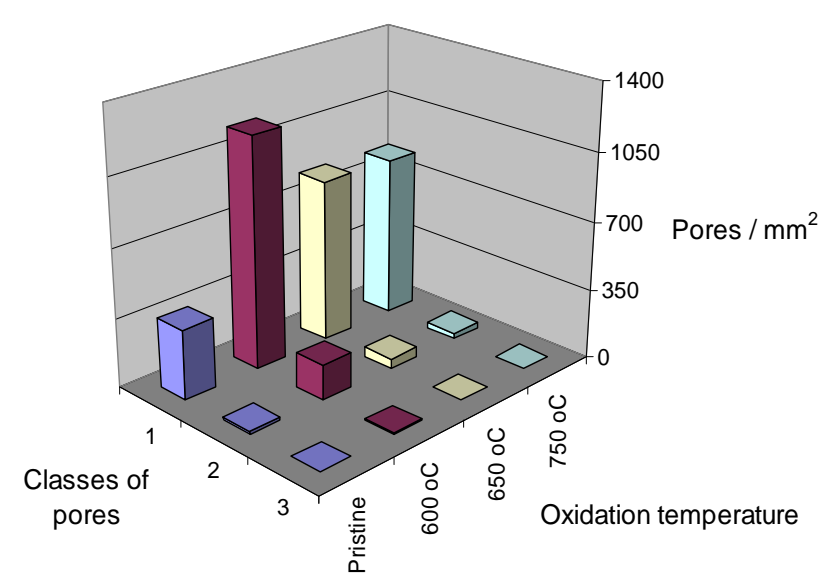

Oxidized PCEA - $10 \%$ weight loss

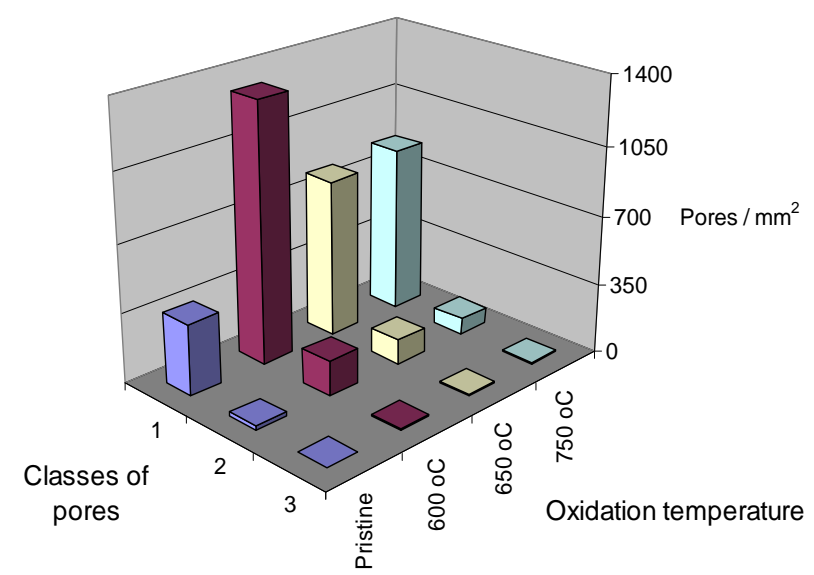

Oxidized PCEA - $20 \%$ weight loss

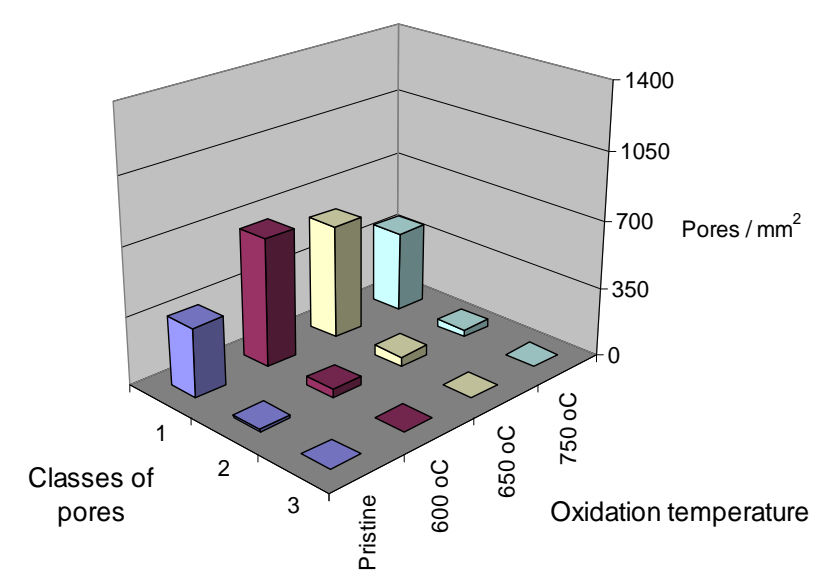

Fig. 18: Pore density distribution in oxidized specimens versus temperature and weight loss level. Classes of pores are defined in Table 2. 
A different conclusion emerges from Figure 18, which shows the distribution of pore density (pores $/ \mathrm{mm}^{2}$ ) in the three classes of pores at various oxidation levels and temperatures. Compared with the pore density of the pristine sample, oxidized samples have larger pore densities at all levels of oxidation and all temperatures. The largest increase is seen for fine pores at low oxidation temperatures $\left(600-650{ }^{\circ} \mathrm{C}\right)$ and low levels of oxidation (up to $15 \%$ ). For specimens oxidized in these conditions, the density of fine pores is up to 4 times (or more) larger than the density of the same size pores in the unoxidized specimen. The increase in the density of medium size pores (class 2) is also significant (compared with the unoxidized sample). As the oxidation temperature increases $\left(750{ }^{\circ} \mathrm{C}\right)$ the density of fine pores decreases in comparison with the same size pores on specimens oxidized at lower temperatures. Significantly, the distribution of pore density on specimens oxidized to $20 \%$ weight loss resembles that of the unoxidized specimen, with a notably close similarity for the specimen oxidized at $750{ }^{\circ} \mathrm{C}$. This finding is in close agreement with the oxidation mechanism at $750{ }^{\circ} \mathrm{C}$, where oxidation causes surface corrosion and dimensional shrinkage, but leaves the core basically unaltered.

In conclusion, optical microscopy combined with image analysis confirms that oxidation at low temperatures spreads almost uniformly throughout the volume of the specimens, and produces no apparent external damage. On the other hand, oxidation at high temperatures causes substantial damage in a narrow surface band, accompanied by erosion and dimensional changes, but leaves the interior of the specimens with little or no changes in the pore structure. 


\section{DISCUSSION: OXIDATION EFFECTS ON MECHANICAL STRENGTH}

There is a definite need of more experimental data on the effect of pore development caused by thermal oxidation on the mechanical properties of graphite [16]. This knowledge is important for prediction of mechanical strength changes caused by development of porosity during oxidation, and for evaluation of integrity and lifetime of HGTR graphite components in real operation conditions.

Several experimental techniques are available for evaluation of porosity in graphite. Among them, mercury intrusion porosimetry and gas adsorption techniques are limited to open pores only. They provide the average distribution (by size) of pores, but are not specific to spatial location of pores in the oxidized material. Other methods based on indirect measurements of density distribution (X-ray tomography, acoustic microscopy) provide maps of both open and closed porosity, but require extensive calibration against standards. Direct density measurements (by layer peeling of oxidized specimens) are tedious and do not give microstructural information on porosity.

Direct microscopic techniques - optical microscopy (at low and medium magnification) and electron microscopy (at high and very high magnification) - afford direct observation of porosity in graphite and of the changes induced by oxidation. In order to derive statistically significant results from these observations, microscopy techniques must be used in combination with automated image analysis. Together, these combined techniques are ideal for mapping the spatial distribution of pores and for obtaining statistical information on their size, shape, orientation etc as well. Such information might later be used for development of finite element analysis models of reaction and diffusion of the oxidant gas in the graphite microstructure, thus allowing a full and detailed description of the microscale phenomena that accompany oxidation of graphite. In a next stage, modeling of porosity development in the oxidation process could be appended to the existing models of crack development and mechanical failure under external stress.

Analysis of porosity development during oxidation of graphite is in a preliminary stage at this juncture. The results and observations reported here confirm the usefulness of optical microscopy enhanced with AIA technique for observation of oxidant penetration during oxidation at various conditions, and of the distribution of oxidized zones between the surface and the bulk regions of graphite specimens. Although on different grades of nuclear graphite, the results reported here for PCEA graphite correlate well with previous observations from the ORNL group [10] on development of non-uniform density profiles in oxidized specialty grade graphite (R4-650). The results are also in very good agreement with recent theoretical estimates advanced by ORNL researchers $[13,14]$ on penetration depth of the oxidant gas at quasi-steady state oxidation conditions. These results confirm one more time the validity of the chemically reactive oxidation zone proposed by Wichner and Ball (ORNL) [17].

Based on the preliminary results presented above, it is apparent that the temperature of oxidation, along with the degree of weight loss, plays an important role on the apparent damage of mechanical properties upon oxidation. The differences are very well illustrated in Fig. 19 which shows four nuclear graphite specimens (in this case, NBG-18 graphite) oxidized at different temperatures to $15 \%$ weight loss (reproduced from [18]) 


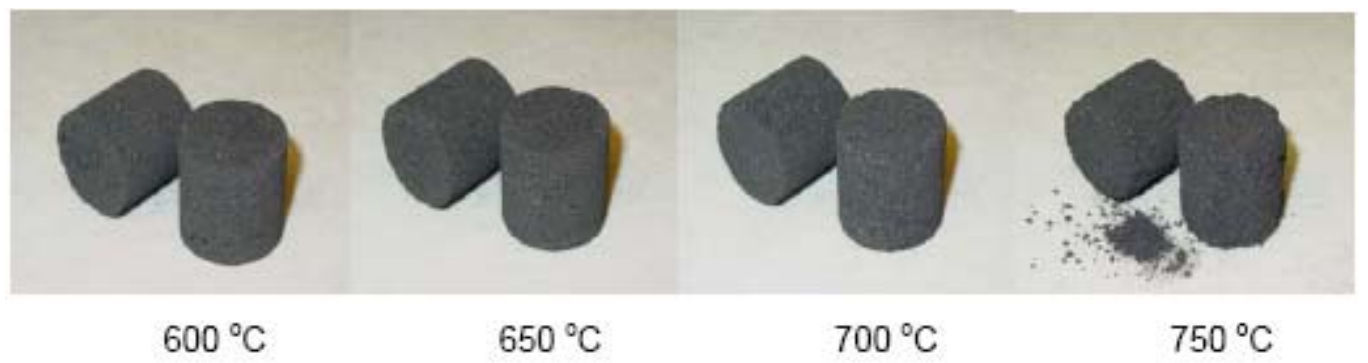

Fig. 19: Nuclear graphite (NBG-18 grade) oxidized at $15 \%$ weight loss and different temperatures. Two specimens were oxidized at each temperature. The size of specimens before oxidation was $25.4 \mathrm{~mm}$ diameter x $25.4 \mathrm{~mm}$ length (Reproduced from [18]) The dust seen after oxidation at $750{ }^{\circ} \mathrm{C}$ is caused by surface corrosion caused by preferential oxidation of the binder.

When the temperature of oxidation is high (above some $700-750{ }^{\circ} \mathrm{C}$ for oxidation in air) the reaction is fast and oxidation is limited to surface corrosion in a narrow layer. This causes local dimensional and shape changes, but the oxidant does not penetrates deep in the bulk of graphite parts. Oxidation in this regime, limited by in-pore diffusion or mass transfer in the boundary layer, is expected not to affect the mechanical strength of large graphite block; however, because reaction is fast, prolonged exposure may cause significant dimensional reduction and structural damage.

On the other hand, oxidation at low temperatures is much slower, but the penetration of the oxidant is deeper. In the oxidized zone, which extends deeper in the mass of graphite, development of fine pores contributes to degradation of mechanical strength of the material. Referring to the Burchell fracture model [4], two mechanisms may account for that: On one hand, opening of fine porosity in the oxidized graphite is expected to lower the critical value of the stress intensity factor, which is a specific property of the basic material (graphite particles) related to the amount of stress that can be applied before failure. On the other hand, the increase in the pore density by itself will favor initiation and propagation of defects. At equivalent degrees of weight loss, it is expected that graphite parts oxidized at low temperature have weaker mechanical strength than the surface-only corroded parts that have been oxidized at high temperatures. In the latter case the preferential oxidation of the binder causes visible surface damage and even some carbon dust (Fig. 19). 


\section{CONCLUSION}

Although in an incipient stage, the present study shows that the automatic image analysis technique applied to optical microscopy is a valuable tool for determination of porosity development in oxidized graphite. The method is quick, affordable, and convenient, and provides quantitative information at a statistically significant level.

In this study, a series of PCEA nuclear graphite specimens was oxidized at various weight loss levels and different temperatures. The oxidized specimens were sectioned and the cross section surface was examined by optical microscopy. Automated image analysis allowed identification of pores in the graphite structure based on their light reflectance properties. The newly created pores (identified as optical objects) were then analyzed on a statistical basis. The results agree qualitatively with the density profile measurements on oxidized graphite samples obtained by direct peeling of graphite, and with the theoretical predictions on penetration of oxidant as a function of temperature. The results demonstrate that uniform oxidation (at low temperatures) is accompanied by development of fine porosity (pores under $100 \mu^{2}$ area), while larger pores seem not to be sensibly affected. This information is useful for understanding the mechanism of pore development during oxidation and the consequences on the mechanical strength of oxidized graphite.

The AIA method should be further standardized and consolidated. The study of porosity development in graphite must be continued with multilevel statistical analysis of pore structures development as a function of weight loss and temperature. AIA technique allows for determination of numerous structural parameters of the pore network, such as orientation, shape, perimeter, location, connectivity, maximum and minimum length of pores etc. A consistent procedure for identification of these properties based on optical images must be defined. Observations at higher magnification (and with improved resolution) are also needed in order to differentiate between the properties of pores (size, shape, location, orientation etc) in the bulk and next to the surface of oxidized specimens. In addition, the use of other characterization techniques (X-ray diffraction, Raman microscopy) may reveal local details of the oxidation mechanism, for example by distinguishing between oxidized graphite filler and the matrix binder in the oxidized zones. For consolidation of the AIA technique, porosity characterization by the optical method should be complemented with quantitative information obtained from well established benchmark techniques for pore characterization, such as gas adsorption (BET method) and mercury intrusion porosimetry.

In conclusion, the results obtained in this preliminary, exploratory work demonstrate the usefulness of optical microscopy enhanced with AIA techniques for analysis of porosity development upon oxidation of graphite. The results correlate with direct observations from direct density measurements. On the long run, the method has the potential to provide microstructural information needed for better understanding and modeling of graphite oxidation effects on the structural integrity of nuclear graphite. 


\section{REFERENCES}

1 Murdie N, Edwards IAS, Marsh $\mathrm{H}$, Changes in porosity of graphite caused by radiolytic gasification by carbon dioxide, Carbon 24 (1986) 267-275.

2 Pickup IM, McEnaney B, Cooke RG, Fracture processes in graphite and the effects of oxidation, Carbon 24 (1986) 535-543.

3 Eto M, Growock FB, Effect of oxidizing environment on the strength of H451, PGX and IG-11 graphites, Carbon 21 (1983) 135-147.

4 Burchell TD, A microstructurally based fracture model for polygranular graphites, Carbon 34 (1996) 297-316

5 Burchell TD, Yahr T, Battiste R, Modeling the multiaxial strength of H-451 nuclear grade graphite, Carbon 45 (2007) 2570-83.

6 Babout L, Mummery PM, Marrow TJ, Tzelepi A, Withers PJ, The effect of thermal oxidation on polycrystalline graphite studied by X-ray tomography, Carbon 43 (2005) 765-774.

7 Babout L, Marrow TJ, Mummery PM, Withers PJ, Mapping the evolution of density in 3D of thermally oxidized graphite for nuclear applications, Carbon 54 (2006) 829-834

8 Fuller EL, Okoh JM, Kinetics and mechanisms of the reaction of air with nuclear grade graphites: IG-110, J Nucl Mater 240 (1997) 241

9 Propp WA, Graphite oxidation: Thermodynamics / reaction, DOE/SNF/REP-018, INEEL, Idaho Falls (Sept. 1998).

10 Contescu CI, Azad S, Miller D, Lance MJ, Baker FS, Practical aspects for characterizing air oxidation of graphite, J Nucl Mater 381 (2008) 15-24

11 Kim ES, Lee WW, No HC, Analysis of geometrical effects on graphite oxidation through measurement of internal surface area, J Nucl Mater 348 (2006) 174-180

12 Chi S-H, Burchell TD, Contescu CI, Density change of an oxidized nuclear graphite by acoustic microscopy and image processing J Eng Gas Turbines Power, 131 (2009) 052904

13 Wichner RP, Burchell TD, Contescu CI, Note on graphite oxidation by oxygen and moisture, ORNL/TM-2008/230, Oak Ridge, TN (November 2008)

14 Wichner RP, Burchell TD, Contescu CI, Penetration depth and transient oxidation of graphite by oxygen and water vapor, J Nucl Mater 393 (2009) 518-521

15 Contescu CI, Burchell TD, ASTM activities on standard method for graphite oxidation, ORNL/GEN4/LTR-09-002, Oak Ridge, TN (August 2009)

16 Berre C, Fok SL, Mummery PM, Ali J, Marsden BJ, Marrow TJ, Neighbour GB, Failure analysi of the effects of porosity in thermally oxidized nuclear graphite using finite element modeling, $\mathrm{J}$ Nucl Mater 381 (2008) 1-8.

17 Wichner RP, Ball SJ, Potential damage to gas-cooled graphite reactors due to severe accidents, ORNL/TM-1366, Oak Ridge, TN (April 1999)

18 Contescu CI, Wichner RP, Guldan T, Oxidation resistance of NGNP graphite materials, ORNL internal report , NGNP Program, Oak Ridge, TN (August 2008) 